\title{
Single-peaked choice
}

\author{
Walter Bossert • Hans Peters
}

Received: 27 August 2007 / Accepted: 30 May 2008 / Published online: 24 June 2008 (C) The Author(s) 2008

\begin{abstract}
Single-peaked preferences have played an important role in the literature ever since they were used by Black (J Polit Econ 56:23-34, 1948) to formulate a domain restriction that is sufficient for the exclusion of cycles according to the majority rule. In this paper, we approach single-peakedness from a choice-theoretic perspective. We show that the well-known axiom of independence of irrelevant alternatives (a form of contraction consistency) and a continuity requirement characterize a class of singlepeaked choice functions. Moreover, we examine rationalizability and representability of these choice functions.
\end{abstract}

Keywords Single-peakedness · Choice functions · Rationalizability · Representability

JEL Classification $\quad \mathrm{D} 11 \cdot \mathrm{D} 71$

\begin{abstract}
We thank Hervé Moulin, John Weymark, and an anonymous referee for comments. Financial support from the Research Institute CIREQ of the Universite de Montréal, the Research School METEOR of the University of Maastricht and the Social Sciences and Humanities Research Council of Canada is gratefully acknowledged.
\end{abstract}

W. Bossert

Département de Sciences Economiques and CIREQ, Université de Montréal, C.P. 6128, succursale Centre-ville, Montréal, QC H3C 3J7, Canada e-mail: walter.bossert@umontreal.ca

H. Peters $(\bowtie)$

Department of Quantitative Economics, University of Maastricht, P.O. Box 616, 6200 MD Maastricht, The Netherlands e-mail: h.peters@ke.unimaas.nl 


\section{Introduction}

Single-peaked preferences have played an important role in the literature ever since they were used by Black (1948) to formulate a domain restriction that is sufficient for the exclusion of cycles according to the majority rule; see also Inada (1969) and Sen (1970) for early contributions that employ domain assumptions of that nature. An example for more recent applications is the area devoted to the study of strategy-proofness, where the single-peakedness restriction on preferences has proven to allow for several classes of possible collective choice rules in one-dimensional policy spaces; see, for example, Moulin (1980) and Sprumont (1991). Single-peakedness (or more specific notions such as spatial or Euclidean preferences) can be defined in higher dimensions as well; see, for instance, Le Breton and Weymark (2007) for a detailed discussion. They arise naturally in many economic models, e.g., by maximizing a strictly quasi-concave utility function on a linear budget set in consumer theory. See also Barberà et al. (1993), Barberà and Jackson (1994), Ehlers and Storcken (2002), and Dutta et al. (2002), to name but a few.

In this paper, we approach single-peakedness from a choice-theoretic perspective. The universal set of alternatives is represented by a Euclidean space (of fixed but arbitrary dimension), and a choice function assigns a unique alternative to each feasible set within the domain of this function. We assume that the domain consists of all non-empty, compact and convex subsets of the Euclidean space, an assumption that is standard in the context of choice in economic environments. Nevertheless, all our results would go through on (for instance) the restricted domain of all polytopes, i.e., sets that are the convex hulls of finitely many points. We comment on domain restrictions in the concluding section.

The central conditions we impose on a choice function are the well-known axiom of independence of irrelevant alternatives, and continuity (with respect to the Hausdorff metric on compact sets). Independence of irrelevant alternatives, IIA, is a form of contraction consistency introduced by Nash (1950) in the context of bargaining problems; it generalizes the Weak Axiom of Revealed Preference from consumer demand theory. Indeed, our approach is based on the idea of revealed preference. We show, in Sect. 3, that IIA and a weaker continuity condition-continuity restricted to intervals on the same straight line - characterize a class of choice functions that we call singlepeaked. A choice function is single-peaked if its direct revealed preference relation is antisymmetric and satisfies the following condition: if an alternative $x$ is direct revealed preferred to another alternative $y$, then it is direct revealed preferred to all alternatives on the straight half-line from $x$ through $y$. This definition implies that such a choice function has at most one (global) peak-a point that is always chosen when it is available. Even if there is no global peak, preference along any straight line decreases monotonically away from a (local) peak on that line, where this peak may also be located at 'infinity'. If a (global) peak does not exist or cannot be chosen, then always a boundary point of the choice set is chosen.

The remainder of the paper is devoted to the issues of rationalizability and representability of single-peaked choice functions. In Sect. 4 we show that if the dimension is at least 2 , then for any natural number $n>2$ there are choice functions with a peak that satisfy IIA and (full) continuity, such that the associated revealed preference 
relation has cycles of length $n$ but no shorter cycles. ${ }^{1}$ Also for choice functions without a peak, IIA and continuity do not guarantee acyclicity of the revealed preference relation, with one exception: if the dimension is 2, then IIA and (the weaker) continuity (condition) imply acyclicity.

In Sect. 5 we show that a continuous choice function is representable by a utility function if the associated revealed preference relation is acyclic, and that such a utility function must be strictly quasi-concave. These results are in line with what is known from demand theory_-for example, Debreu (1972) — about continuity of preferences and convexity of choice sets, but they nevertheless require proofs and to the best of our knowledge are new.

Again to the best of our knowledge, our results are original for dimension at least 2. Of course, they are related to other work in the area of choice and demand, (revealed) preference, and rationalizability-representability: the directly relevant sources will be mentioned as the paper develops. Moulin (1984) examines social choice functions defined on a domain involving single-peaked preference profiles that satisfy suitably formulated versions of independence properties. As a by-product of his analysis he obtains a characterization of single-peaked choice from closed intervals within $[0,1]$ based on IIA and continuity (see Remark 1 in Moulin 1984). This is closely related to our results, applied to the one-dimensional case, where every single-peaked choice function is rationalizable and representable.

Ballester and Haeringer (2006) provide a characterization of (one-dimensional) single-peaked preference profiles. They examine the question under what conditions there exists a single ranking of the alternatives such that all preferences within the profile are single-peaked with respect to this ranking. Thus, although the title of their paper may suggest differently, their work is not closely related to our work.

The outline of the paper has been given above. Some concluding remarks are collected in Sect. 6.

\section{Preliminaries}

We consider choices from sets of $k$-dimensional vectors, where $k \in \mathbb{N}$ is arbitrary but fixed. For a non-empty set $C \subseteq \mathbb{R}^{k}, \operatorname{conv}(C)$ denotes the convex hull of $C$, and $\operatorname{bd}(C)$ denotes the (topological) boundary of $C$. If $C=\{x, y\}$ for some $x, y \in \mathbb{R}^{k}$, we also write $[x, y]$ instead of $\operatorname{conv}(C)$ and refer to this set as an interval. The (relatively) open or half-open sets $(x, y],[x, y)$, and $(x, y)$ are defined in the obvious way. For distinct $x, y \in \mathbb{R}^{k},[x, y, \rightarrow)$ denotes the half-line through $y$ starting at $x$ and $\ell(x, y)$ denotes the straight line through $x$ and $y$.

For a (binary) relation $R \subseteq \mathbb{R}^{k} \times \mathbb{R}^{k}$, we use $P$ to denote the asymmetric part of $R$, that is, $x P y$ if and only if $x R y$ and $\neg y R x$ for all $x, y \in \mathbb{R}^{k}$. A relation $R$ on $\mathbb{R}^{k}$ is: (i) reflexive if $x R x$ for all $x \in \mathbb{R}^{k}$; (ii) complete if $x R y$ or $y R x$ for all $x, y \in \mathbb{R}^{k}$ such that $x \neq y$; (iii) transitive if $\left[x R y\right.$ and $y R z$ ] implies $x R z$ for all $x, y, z \in \mathbb{R}^{k}$;

\footnotetext{
${ }^{1}$ The construction of these choice functions involves some interesting classical geometry, see also the Appendix to the paper. Moreover, so far we have not been able to find simpler choice functions to do the work.
} 
(iv) antisymmetric if $[x R y$ and $y R x]$ implies $x=y$ for all $x, y \in \mathbb{R}^{k} ;(\mathrm{v})$ acyclic if there exist no $m \in \mathbb{N} \backslash\{1,2\}$ and $x^{1}, \ldots, x^{m}$ such that $x^{i} P x^{i+1}$ for all $i \in\{1, \ldots, m\}$, where $x^{m+1}:=x^{1}$.

The set of all non-empty, compact and convex subsets of $\mathbb{R}^{k}$ is denoted by $\mathcal{C}$. The convergence of a sequence in $\mathcal{C}$ is defined in terms of the Hausdorff metric. ${ }^{2}$

A choice function is a mapping $\varphi: \mathcal{C} \rightarrow \mathbb{R}^{k}$ such that $\varphi(C) \in C$ for all $C \in \mathcal{C}$. So a choice function is single-valued, and it is defined for every non-empty, compact and convex subset of $\mathcal{C}$, including single points. A choice function $\varphi$ on $\mathcal{C}$ induces a relation $R_{\varphi}$ on $\mathbb{R}^{k}$ defined by

$$
x R_{\varphi} y: \Leftrightarrow \text { there exists } C \in \mathcal{C} \text { such that } y \in C \text { and } \varphi(C)=x
$$

for all $x, y \in \mathbb{R}^{k}$. The relation $R_{\varphi}$ is called the direct revealed preference relation corresponding to the choice function $\varphi$. Due to our domain assumption (in particular, because $\{x\} \in \mathcal{C}$ for all $\left.x \in \mathbb{R}^{k}\right), R_{\varphi}$ is reflexive. The (indirect) revealed preference relation $\overline{R_{\varphi}}$ corresponding to $\varphi$ is the transitive closure of $R_{\varphi}$, that is,

$$
\begin{gathered}
x \overline{R_{\varphi}} y: \Leftrightarrow \quad \text { there are } m \in \mathbb{N} \backslash\{1\} \quad \text { and } x^{1}, \ldots, x^{m} \in \mathbb{R}^{k} \text { such that } \\
x=x^{1}, \quad x^{i} R x^{i+1} \forall i \in\{1, \ldots, m-1\} \quad \text { and } \quad x^{m}=y
\end{gathered}
$$

for all $x, y \in \mathbb{R}^{k}$.

The following properties of a choice function $\varphi: \mathcal{C} \rightarrow \mathbb{R}^{k}$ are of importance in this paper.

Independence of Irrelevant Alternatives (IIA). For all $C, D \in \mathcal{C}$,

$$
C \subseteq D \quad \text { and } \quad \varphi(D) \in C \Rightarrow \varphi(C)=\varphi(D)
$$

IIA is the standard contraction-independence property for single-valued choice functions; see Nash (1950) for its application in a bargaining framework.

Continuity (CON). For all $C \in \mathcal{C}$ and for all sequences $\left\langle C^{i}\right\rangle_{i \in \mathbb{N}}$ with $C^{i} \in \mathcal{C}$ for all $i \in \mathbb{N}$,

$$
\lim _{i \rightarrow \infty} C^{i}=C \Rightarrow \lim _{i \rightarrow \infty} \varphi\left(C^{i}\right)=\varphi(C)
$$

We also use the following weaker version of continuity. In this weakening the continuity axiom applies to intervals along the same line only.

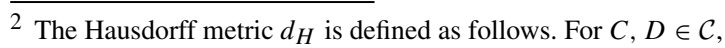

$$
d_{H}(C, D)=\max \left\{\max _{c \in C}\left\{\min _{d \in D}\|d-c\|\right\}, \max _{d \in D}\left\{\min _{c \in C}\|d-c\|\right\}\right\},
$$

where $\|\cdot\|$ denotes the Euclidean metric. 
Collinear Interval Continuity (CIC). For all distinct $x, y \in \mathbb{R}^{k}$ and for all sequences $\left\langle x^{i}\right\rangle_{i \in \mathbb{N}}$ and $\left\langle y^{i}\right\rangle_{i \in \mathbb{N}}$ with $x^{i}, y^{i} \in \ell(x, y)$ for all $i \in \mathbb{N}$,

$$
\lim _{i \rightarrow \infty} x^{i}=x \quad \text { and } \quad \lim _{i \rightarrow \infty} y^{i}=y \Rightarrow \lim _{i \rightarrow \infty} \varphi\left(\left[x^{i}, y^{i}\right]\right)=\varphi([x, y]) .
$$

If $\varphi$ satisfies IIA, then $x R_{\varphi} y$ implies $\neg y R_{\varphi} x$ for all $x, y \in \mathbb{R}^{k}$ such that $x \neq y$ : this is so since, under IIA, $x R_{\varphi} y$ is equivalent to $\varphi([x, y])=x$. Thus, if $\varphi$ satisfies IIA, then the direct revealed preference relation $R_{\varphi}$ is antisymmetric.

We proceed with definitions of the well-known notions of rationalizability and rationalizability-representability of a choice function, formulated for our specific environment. A choice function $\varphi: \mathcal{C} \rightarrow \mathbb{R}^{k}$ is rationalizable if there exists a transitive relation $R$ on $\mathbb{R}^{k}$ such that

$$
\{\varphi(C)\}=\{x \in C \mid x R y \text { for all } y \in C\}
$$

for all $C \in \mathcal{C} .^{3}$ A choice function $\varphi$ is rationalizable-representable if there exist a transitive relation $R$ on $\mathbb{R}^{k}$ and a function $u: \mathbb{R}^{k} \rightarrow \mathbb{R}$ such that $R$ rationalizes $\varphi$ and

$$
[x R y \Rightarrow u(x) \geq u(y)] \quad \text { and } \quad[x P y \Rightarrow u(x)>u(y)]
$$

for all $x, y \in \mathbb{R}^{k}$.

A function $u: \mathbb{R}^{k} \rightarrow \mathbb{R}$ is strictly quasi-concave if for all $z, z^{\prime} \in \mathbb{R}^{k}$ with $z \neq z^{\prime}$ and all $0<\alpha<1, u\left(\alpha z+(1-\alpha) z^{\prime}\right)>\min \left\{u(z), u\left(z^{\prime}\right)\right\}$.

\section{Single-peaked choice functions}

The objective of this paper is to study choice functions that satisfy IIA and continuity $(\mathrm{CON})$. We will show that such choice functions are single-peaked in the sense described by the following definition.

Definition 3.1 A choice function $\varphi: \mathcal{C} \rightarrow \mathbb{R}^{k}$ is single-peaked if

(i) $R_{\varphi}$ is antisymmetric;

(ii) for all $x, y \in \mathbb{R}^{k}$ with $x \neq y$,

$$
x R_{\varphi} y \Rightarrow x R_{\varphi} z \quad \text { for all } \quad z \in[x, y, \rightarrow) .
$$

Thus, a choice function is single-peaked if (i) the direct revealed preference relation is antisymmetric and (ii) for all distinct $x$ and $y$, if $x$ is directly revealed preferred to $y$ then $x$ is directly revealed preferred to any point on the straight half-line through $y$ emanating from $x$.

Note that the definition of single-peakedness implies the following. If $x$ is directly revealed preferred to $y \neq x$, then for any two points $z \neq z^{\prime}$ on the straight half-line

\footnotetext{
3 Requiring the rationalizing relation to be reflexive, complete and transitive leads to an equivalent formulation of rationalizability; see Richter $(1966,1971)$.
} 
emanating from $x$ through $y$, if $z$ is closer to $x$ than $z^{\prime}$ is, then $z$ is directly revealed preferred to $z^{\prime}$. This follows from the fact that $\varphi\left(\left[z, z^{\prime}\right]\right)=z$, since otherwise we would have $\varphi\left(\left[z, z^{\prime}\right]\right) R_{\varphi} z$, hence $\varphi\left(\left[z, z^{\prime}\right]\right) R_{\varphi} x$ by applying (ii) in the definition of single-peakedness, a contradiction.

For later reference we formulate this observation as a proposition.

Proposition 3.2 Let $\varphi: \mathcal{C} \rightarrow \mathbb{R}^{k}$ be a single-peaked choice function. If $x \neq y$ and $x R_{\varphi} y$, then $z R_{\varphi} z^{\prime}$ for all $z^{\prime} \in[x, y, \rightarrow)$ and $z \in\left[x, z^{\prime}\right]$.

The expression 'single-peaked' is motivated by the following observation. Let the choice function $\varphi$ be single-peaked and suppose that there is a $C \in \mathcal{C}$ such that $p:=\varphi(C)$ is an interior point of $C$. Since, for any point $x \in \mathbb{R}^{k}$, the half-line $[p, x, \rightarrow)$ contains a point of $C$ distinct from $p$, single-peakedness implies that $p P_{\varphi} x$. Hence, $p$ is directly revealed strictly preferred to every $x \in \mathbb{R}^{k}$. This implies $\varphi(D)=p$ for every $D \in \mathcal{C}$ with $p \in D$. Moreover, for every other $D \in \mathcal{C}, \varphi(D)$ must be a boundary point. Summarizing, we have the following proposition.

Proposition 3.3 Let $\varphi: \mathcal{C} \rightarrow \mathbb{R}^{k}$ be a single-peaked choice function. Then either $(i)$ or (ii) holds, where:

(i) There is a $p \in \mathbb{R}^{k}$ such that for all $C \in \mathcal{C}, \varphi(C)=p$ if $p \in C$ and $\varphi(C) \in \operatorname{bd}(C)$ if $p \notin C$.

(ii) $\varphi(C) \in \operatorname{bd}(C)$ for all $C \in \mathcal{C}$.

If case (i) applies in Proposition 3.3, the point $p$ is called a peak of $\varphi$. Clearly, if a single-peaked choice function has no peak in the sense of (i), then nevertheless it still induces a 'local' peak (possibly at 'infinity') on each straight line.

For $k=1$, this definition reduces to rationalizability by single-peaked preferences: if there is a peak $p \in \mathbb{R}$, define the relation $R$ by letting

$$
x R y: \Leftrightarrow|x-p| \leq|y-p|
$$

for all $x, y \in \mathbb{R}$; if there is no peak, let either

$$
x R y: \Leftrightarrow x \leq y
$$

for all $x, y \in \mathbb{R}$ or

$$
x R y: \Leftrightarrow x \geq y
$$

for all $x, y \in \mathbb{R}$, whichever case applies. Moreover, rationalizability-representability is guaranteed: for the three possibilities illustrated above, the corresponding utility function $u: \mathbb{R} \rightarrow \mathbb{R}$ can be defined by

$$
u(x)=-|x-p|
$$

for all $x \in \mathbb{R}$ or

$$
u(x)=-x
$$


for all $x \in \mathbb{R}$ or

$$
u(x)=x
$$

for all $x \in \mathbb{R}$, respectively. ${ }^{4}$

Single-peaked choice functions are characterized by IIA and CIC, as established in the following theorem.

Theorem 3.4 Let $k \in \mathbb{N}$. A choice function $\varphi: \mathcal{C} \rightarrow \mathbb{R}^{k}$ satisfies IIA and CIC if and only if $\varphi$ is single-peaked.

Proof 'If.' Suppose $\varphi$ is single-peaked.

To establish IIA, suppose that $C, D \in \mathcal{C}$ with $C \subseteq D$ and $\varphi(D) \in C$. If $\varphi(C) \neq$ $\varphi(D)$, then by definition of $R_{\varphi}$, both $\varphi(C) R_{\varphi} \varphi(D)$ and $\varphi(D) R_{\varphi} \varphi(C)$, a contradiction with part (i), antisymmetry of $R_{\varphi}$. Hence, $\varphi(C)=\varphi(D)$ and IIA holds.

To prove that CIC is satisfied, let $x, y$ be distinct elements of $\mathbb{R}^{k}$ and consider sequences $\left\langle x^{i}\right\rangle_{i \in \mathbb{N}}$ and $\left\langle y^{i}\right\rangle_{i \in \mathbb{N}}$ with $x^{i}, y^{i} \in \ell(x, y)$ for all $i \in \mathbb{N}$, and with $\lim _{i \rightarrow \infty} x^{i}=x$ and $\lim _{i \rightarrow \infty} y^{i}=y$. It is sufficient to show that any convergent subsequence of $\left(\varphi\left(\left[x_{i}, y_{i}\right]\right)\right)_{i \in \mathbb{N}}$ has limit $\varphi([x, y])$. Without loss of generality suppose $\lim _{i \rightarrow \infty} \varphi\left(\left[x^{i}, y^{i}\right]\right)$ exists. To the contrary, suppose that $v:=\varphi([x, y]) \neq$ $\lim _{i \rightarrow \infty} \varphi\left(\left[x^{i}, y^{i}\right]\right)=: w \in[x, y]$. Then $v R_{\varphi} w$, (without loss of generality) $\varphi\left(\left[x^{i}, y^{i}\right]\right) \neq v$ for all $i$, and by part (ii) of single-peakedness, $v R_{\varphi} \varphi\left(\left[x^{i}, y^{i}\right]\right)$ for all $i$. If $w \in(x, y)$ then we can choose $i$ such that $\varphi\left(\left[x^{i}, y^{i}\right]\right) \in\left(x^{i}, y^{i}\right)$, so that $\varphi\left(\left[x^{i}, y^{i}\right]\right) R_{\varphi} x^{i}$ and $\varphi\left(\left[x^{i}, y^{i}\right]\right) R_{\varphi} y^{i}$, and hence by (ii), we obtain $\varphi\left(\left[x^{i}, y^{i}\right]\right) R_{\varphi} v$, a contradiction with antisymmetry of $R_{\varphi}$. If $w \notin(x, y)$ then without loss of generality $w=y$; then $v \in[x, y)$ and we can choose $i$ such that $\varphi\left(\left[x^{i}, y^{i}\right]\right) \in\left(v, y^{i}\right]$, so that $\varphi\left(\left[x^{i}, y^{i}\right]\right) R_{\varphi} v$ by (ii), again a contradiction with antisymmetry. This completes the proof of CIC of $\varphi$.

'Only if.' Suppose $\varphi$ satisfies IIA and CIC.

As remarked earlier, antisymmetry of $R_{\varphi}$ follows from IIA. To prove part (ii) of single-peakedness, let $x, y$ be distinct elements of $\mathbb{R}^{k}$ with $x R_{\varphi} y$ and let $z \in$ $[x, y, \rightarrow)$. Assume, contrary to what we wish to prove, $\neg x R_{\varphi} z$. Let $v:=\varphi([x, z])$, then $v \neq x$ and by IIA, $\varphi([x, v])=v$. Also by IIA, $\varphi([x, y])=x$. Hence, again by IIA, $v \in[x, y, \rightarrow) \backslash[x, y]$, and for all $w \in[v, y]$, either $\varphi([x, w])=x$ or $\varphi([x, w]) \in(y, w]$. Let $w_{\beta}:=(1-\beta) y+\beta v$ for every $0 \leq \beta \leq 1$ and $\beta^{*}:=$ $\inf \left\{0 \leq \beta \leq 1 \mid \varphi\left(\left[x, w_{\beta}\right]\right) \in\left[y, w_{\beta}\right]\right\}$. (The infimum exists since $\varphi([x, v])=v$.) Then $\varphi\left(\left[x, w_{\beta}\right]\right)=x$ for all $0 \leq \beta<\beta^{*}$, so by CIC, $\varphi\left(\left[x, w_{\beta^{*}}\right]\right)=x$. But again by CIC, $\varphi\left(\left[x, w_{\beta^{*}}\right]\right) \in\left[y, \beta^{*}\right]$, a contradiction. This completes the proof of part (ii) and of the theorem.

Single-peaked choice functions do not necessarily satisfy full continuity, CON. For instance, the single-peaked choice function picking the lexicographic maximum of a choice set in $\mathbb{R}^{2}$ does not satisfy CON (cf. also Example 5.1). In other words, the class of all IIA and CON choice functions is a proper subclass of the class of single-peaked choice functions.

\footnotetext{
4 As mentioned in the Introduction, a similar result was derived in Moulin (1984) for choice functions defined on subsets of a closed interval. See in particular his Remark 1.
} 


\section{Rationalizability}

If $k=1$, single-peaked choice functions are rationalizable, as mentioned earlier. Thus, IIA and CIC (which is equivalent to $\mathrm{CON}$ in this case) together guarantee rationalizability. However, matters are more complex in higher dimensions, also under (IIA and) $\mathrm{CON}$.

Suppose $k \geq 2$. As noted before, IIA implies that $R_{\varphi}$ is antisymmetric and, thus, there are no cycles of length two in $R_{\varphi}$. Conversely, the absence of cycles of length two in $R_{\varphi}$ implies IIA. Thus, these two conditions are equivalent. Although necessary, IIA (or, equivalently, the absence of cycles of length two) is not sufficient for rationalizability unless specific domain assumptions are made. What is sufficient on any domain is the strong axiom of revealed preference which, in our setting, is equivalent to the requirement that the revealed preference relation $R_{\varphi}$ be acyclic (or, equivalently, that the revealed preference relation $\overline{R_{\varphi}}$ be antisymmetric). This raises the question whether IIA, possibly together with CIC or CON, is sufficient to rule out cycles of arbitrary length on our domain, thus guaranteeing rationalizability. More generally, we investigate whether ruling out cycles of length $n \in \mathbb{N}$ or less is sufficient to rule out longer cycles for arbitrary $n \geq 2$. In answering this question, it turns out that we have to distinguish between the two possibilities (i) and (ii) in Proposition 3.3.

\subsection{Choice functions with a peak}

In the following example we show that, for any $n \in \mathbb{N}$ with $n \geq 2$, there is a choice function with a peak (in the example the point $\mathbf{0}$ ), satisfying IIA and CON, such that there are no cycles of length $n$ (or smaller) but there are cycles of length $n+1$ (or larger). This implies that, without further assumptions, there is no $n$ such that the exclusion of cycles of length $n$ implies the exclusion of all cycles, for a choice function satisfying IIA and CON.

Example 4.1 Let $k=2$. Let $\alpha \in[0, \pi / 2]$. We are going to construct a choice function $\varphi^{\alpha}$. For $C \in \mathcal{C}$ with $\mathbf{0} \in C$ let $\varphi^{\alpha}(C):=\mathbf{0}$. Now let $C \in \mathcal{C}$ with $\mathbf{0} \notin C$.

For every $\beta \in[0,2 \pi)$ let $\ell_{\beta}$ be the half-line starting from the origin and forming an angle of $\beta$ radians with the positive horizontal axis. Then the set $B(C):=\{\beta \in$ $\left.[0,2 \pi) \mid \ell_{\beta} \cap C \neq \varnothing\right\}$ is either of the form $\left[\beta_{1}, \beta_{2}\right]$ with $0 \leq \beta_{1} \leq \beta_{2}<2 \pi$ or of the form $\left[\beta_{1}, 2 \pi\right) \cup\left[0, \beta_{2}\right]$ with $0 \leq \beta_{2}<\beta_{1}<2 \pi$. For every $\beta \in B(C)$ let $x_{\beta}$ be the point in $C \cap \ell_{\beta}$ closest to the origin. We define the correspondence $c: B(C) \rightarrow[0, \pi / 2]$ as follows: for every $\beta \in B(C), c(\beta)$ is the interval of the non-obtuse angles (in radians) between $\ell_{\beta}$ and those supporting lines of the set $C$ at the point $x_{\beta}$ that (weakly) separate $C$ from the origin. Then $0 \in c\left(\beta_{1}\right)$ and $0 \in c\left(\beta_{2}\right)$. There is a unique value of $\beta$ with $\pi / 2 \in c(\beta)$, namely the value of $\beta$ such that $x_{\beta}$ is the point of $C$ with minimal Euclidean distance to the origin. The correspondence $c$ strictly increases from $\beta_{1}$ to this value, and then strictly decreases again to $\beta_{2}$. In particular, there are at most two different values of $\beta$ such that $\alpha \in c(\beta)$. Let these values be $\beta^{\prime}$ and $\beta^{\prime \prime}$ such that $\beta^{\prime} \bmod \beta_{1} \leq \beta^{\prime \prime} \bmod \beta_{1}$, then $\varphi^{\alpha}(C):=x_{\beta^{\prime}}$.

In other words, if $C$ contains the origin then $\varphi^{\alpha}$ chooses the origin. Otherwise, $\varphi^{\alpha}$ chooses a point from the boundary of $C$ such that there is a supporting line of $C$ at 


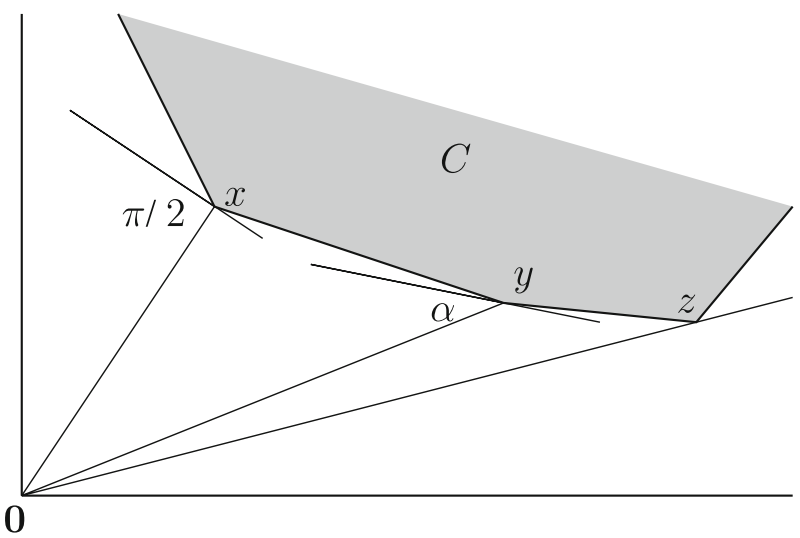

Fig. 1 Illustration of the set of choice functions defined in Example 4.1. The choice set is the shaded set $C$, and $x=\varphi^{\pi / 2}(C), y=\varphi^{\alpha}(C)$ for some value $\alpha$ between 0 and $\pi / 2$, and $z=\varphi^{0}(C)$

this point that forms an angle of $\alpha$ radians with the line through this point and the origin. The chosen point is always the point on the latter line closest to the origin, and in case there are two such points, on different lines through the origin, then $\varphi^{\alpha}$ takes the first one going counter clockwise. For instance, for $\alpha=\pi / 2, \varphi^{\alpha}(C)$ is the point of $C$ closest to the origin. For $\alpha=0$, it takes the point of $C$ closest to the origin on the first supporting line of $C$ passing through the origin when going counter clockwise. See Fig. 1.

For every $\alpha \in[0, \pi / 2]$, the choice function $\varphi^{\alpha}$ is well-defined and satisfies IIA. For every $\alpha \in(0, \pi / 2], \varphi^{\alpha}$ satisfies $\mathrm{CON}$, but for $\alpha=0$ it does not.

We will prove a theorem which implies our earlier claim: that, for every $n \geq 2$, there is a choice function satisfying IIA and CON which has no cycles of length $n$ or smaller, but which has cycles of length $n+1$ or larger. In the proof, we make use of the following result which is proven in the Appendix. For every $n \in \mathbb{N}$ with $n \geq 2$, define

$$
A(n)=\frac{n-2}{2 n} \pi
$$

The number $A(n)$ is equal to half the angle at a vertex in a regular $n$-polygon.

For distinct $x, y, z \in \mathbb{R}^{2}$ we denote by $\measuredangle x y z \in[0, \pi]$ the size (in radians) of the angle formed between the straight line through $x$ and $y$ and the straight line through $z$ and $y$.

Theorem 4.2 Let $k=2$, let $\mathcal{P}$ be a convex $n$-polygon with vertices $x^{1}, \ldots, x^{n}$ and let $\hat{x}$ be a point of $\mathcal{P}$ such that $\measuredangle \hat{x} x^{1} x^{2}=\measuredangle \hat{x} x^{2} x^{3}=\cdots=\measuredangle \hat{x} x^{n-1} x^{n}=: \alpha<\pi / 2$ and $\measuredangle \hat{x} x^{n} x^{1} \geq \alpha$. Then $\alpha \leq A(n)$.

The main result of this subsection is the following theorem.

Theorem 4.3 Let $k=2$, let $\alpha \in(0, \pi / 2)$ and let $n \in \mathbb{N} \backslash\{1\}$ be such that $A(n)<$ $\alpha \leq A(n+1)$. Then $R_{\varphi^{\alpha}}$ has no cycle of length $n$ but it does have a cycle of length $n+1$. 


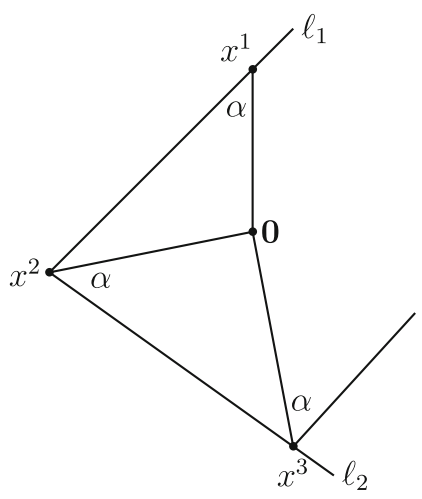

(i)

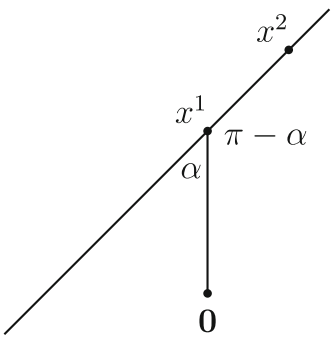

(ii)

Fig. 2 Illustrations for the proof of Theorem 4.3

Proof We first exhibit an $(n+1)$-cycle for $R_{\varphi^{\alpha}}$, and then show that there are no smaller cycles.

To exhibit an $(n+1)$-cycle, take a regular $(n+1)$-polygon with $\mathbf{0}$ as center. Let $x^{1}, \ldots, x^{n+1}$ be the successive vertices of this polygon. Since $\measuredangle \mathbf{0} x^{1} x^{2}=A(n+1) \geq \alpha$, it follows that $\varphi^{\alpha}\left(\left[x^{1}, x^{2}\right]\right)=x^{1}$ by definition of $\varphi^{\alpha}$, hence $x^{1} R_{\varphi^{\alpha}} x^{2}$. This argument can be repeated for any successive pair of vertices, so that $x^{1} R_{\varphi^{\alpha}} x^{2} R_{\varphi^{\alpha}} \ldots R_{\varphi^{\alpha}} x^{n+1}$ $R_{\varphi^{\alpha}} x^{1}$. So $R_{\varphi^{\alpha}}$ has an $(n+1)$-cycle.

Next, we show that there are no smaller cycles. It is sufficient to show that there are no cycles of length $n$. Suppose, to the contrary, that there is such a cycle

$$
x^{1} R_{\varphi^{\alpha}} x^{2} R_{\varphi^{\alpha}} \ldots R_{\varphi^{\alpha}} x^{n} R_{\varphi^{\alpha}} x^{1} .
$$

Obviously, $\mathbf{0}$ is not an element of this cycle since it is the peak of $\varphi^{\alpha}$. Furthermore, we may assume that for all $i, j \in\{1, \ldots, n\}, x^{i} R_{\varphi^{\alpha}} x^{j}$ implies that either $i<n$ and $j=i+1$ or $i=n$ and $j=n+1$ since otherwise we would have an even shorter cycle with $\ldots R_{\varphi^{\alpha}} x^{i} R_{\varphi^{\alpha}} x^{j} R_{\varphi^{\alpha}} \ldots$ by leaving out the points between $x^{i}$ and $x^{j}$ in the original cycle.

Consider $x^{1}$ and $x^{2}$. Since $x^{1} R_{\varphi^{\alpha}} x^{2}, x^{2}$ is a point separated from $\mathbf{0}$ by the line $\ell_{1}$ passing through $x^{1}$ and forming an angle of $\alpha$ radians with the line through $\mathbf{0}$ and $x^{1}$. Suppose $x^{2}$ is not on $\ell_{1}$. Since $x^{3}$ must be on the same side of $\ell_{1}$ as $\mathbf{0}$, we can replace $x^{2}$ by the unique point $x^{\prime}$ in $\left[x^{2}, x^{3}\right] \cap \ell_{1}$ and still have $x^{1} R_{\varphi^{\alpha}} x^{\prime} R_{\varphi^{\alpha}} x^{3}$. Hence, we can without loss of generality assume that $x^{2}$ is on $\ell_{1}$. There are two cases to consider, namely (i) $\measuredangle \mathbf{0} x^{1} x^{2}=\alpha$; and (ii) $\measuredangle \mathbf{0} x^{1} x^{2}=\pi-\alpha$.

Case (i): Assume $\measuredangle \mathbf{0} x^{1} x^{2}=\alpha$ and now consider $x^{2}$ and $x^{3}$. By repeating the argument of the previous paragraph, we may assume that $x^{3}$ is a point on the line $\ell_{2}$ passing through $x^{2}$ and forming an angle of $\alpha$ radians with the line through $\mathbf{0}$ and $x^{2}$. We must have $\measuredangle \mathbf{0} x^{2} x^{3}=\alpha$, since $\measuredangle \mathbf{0} x^{2} x^{3}=\pi-\alpha$ would imply that $x^{3}$ would be separated from 0 by $\ell_{1}$ and, thus, $x^{1} R_{\varphi^{\alpha}} x^{3}$, a contradiction. See Fig. 2(i) for the construction so far. 
Repeating this argument up to and including the pair $x^{n-1}$ and $x^{n}$, we have $n-1$ lines $\ell_{1}, \ldots, \ell_{n-1}$, with, for each $i=1, \ldots, n-1, \ell_{i}$ passing through $x^{i}$ and $x^{i+1}$ and such that each $x_{j}(j \in\{1, \ldots, n\} \backslash\{i, i+1\})$ and $\mathbf{0}$ are on the same side of $\ell_{i}$ but not on $\ell_{i}$. Moreover, $\measuredangle \mathbf{0} x^{i} x^{i+1}=\alpha$ for each $i=1, \ldots, n-1$. Hence, $\mathcal{P}:=\operatorname{conv}\left\{x^{1}, \ldots, x^{n}\right\}$ is a convex $n$-polygon with vertices $x^{1}, \ldots, x^{n}$, containing $\mathbf{0}$ as an interior point, such that $\measuredangle \mathbf{0} x^{i} x^{i+1}=\alpha$ for each $i=1, \ldots, n-1$ and $\measuredangle \mathbf{0} x^{n} x^{1} \geq \alpha$. The last inequality follows since $x^{n} R_{\varphi^{\alpha}} x^{1}$. (Observe that we cannot assume equality here: this might involve having to replace $x^{1}$ by a different point $x^{\prime}$ but then $\measuredangle \mathbf{0} x^{\prime} x^{2}>\alpha$.) By applying Theorem 4.2 to $\mathcal{P}$, it follows that $\alpha \leq A(n)$, a contradiction.

Case (ii): Assume $\measuredangle \mathbf{0} x^{1} x^{2}=\pi-\alpha$; see Fig. 2 (ii). Since $\alpha<\pi / 2$, this implies $\left\|x^{2}\right\|>\left\|x^{1}\right\|$ (where $\|\cdot\|$ denotes the Euclidean norm). Since also $\measuredangle \mathbf{0} x^{2} x^{3} \geq \pi-\alpha$ (otherwise we would have $x^{1} R_{\varphi^{\alpha}} x^{3}$, a contradiction), we have $\left\|x^{3}\right\|>\left\|x^{2}\right\|$. Continuing this argument, we obtain $\left\|x^{1}\right\|<\left\|x^{2}\right\|<\cdots<\left\|x^{n}\right\|<\left\|x^{1}\right\|$. This contradiction takes care of case (ii) and completes the proof of the theorem.

Intuitively it is clear that acyclicity of the revealed preference relation is even harder to obtain for higher dimensions. One way to extend Example 4.1 and Theorem 4.3 to $k>2$ may be to 'embed' the choice function $\varphi^{\alpha}$ in higher dimensions, similar to the construction in Peters and Wakker (1994).

\subsection{Choice functions without a peak}

If $\varphi$ is a choice function such that $\varphi(C)$ is on the boundary of $C$ for every feasible set $C$, as in case (ii) in Proposition 3.3, the situation is different. The first observation is that, in this case, cycles of length three cannot occur if $k=2$. Moreover, this observation is a consequence of IIA alone-no continuity requirement is needed.

Lemma 4.4 Let $k=2$. If a choice function $\varphi: \mathcal{C} \rightarrow \mathbb{R}^{k}$ satisfies IIA and $\varphi(C) \in$ $\operatorname{bd}(C)$ for all $C \in \mathcal{C}$, then $R_{\varphi}$ has no cycles of length three.

Proof Suppose, to the contrary, that $x^{1}, x^{2}, x^{3} \in \mathbb{R}^{2}$ are three different points and $x^{1} R_{\varphi} x^{2} R_{\varphi} x^{3} R_{\varphi} x^{1}$. If these three points are not on the same line, then $\varphi\left(\operatorname{conv}\left(\left\{x^{1}, x^{2}\right.\right.\right.$, $\left.\left.\left.x^{3}\right\}\right)\right)$ must be a point on the boundary, i.e., in $\left[x^{1}, x^{2}\right]$ or in $\left[x^{2}, x^{3}\right]$ or in $\left[x^{3}, x^{1}\right]$. In the first case, $\varphi\left(\operatorname{conv}\left(\left\{x^{1}, x^{2}, x^{3}\right\}\right)\right)=x^{1}$ by IIA, but then $x^{1} R_{\varphi} x^{3}$, a contradiction. The other two cases lead to similar contradictions. If the three points are on the same line then an analogous argument applies.

The following theorem shows that adding CIC allows us to extend this result to cycles of arbitrary length.

Theorem 4.5 Let $k=2$. If a choice function $\varphi: \mathcal{C} \rightarrow \mathbb{R}^{k}$ satisfies IIA and CIC and $\varphi(C) \in \operatorname{bd}(C)$ for all $C \in \mathcal{C}$, then $R_{\varphi}$ is acyclic.

Proof The proof proceeds by induction on the cycle length. By IIA, there are no cycles of length two and by Lemma 4.4 there are no cycles of length three. Let $m \geq 4$ and assume as induction hypothesis that there are no cycles of length smaller than $m$. Suppose that $x^{1}, \ldots, x^{m}$ are $m$ different points such that $x^{1} R_{\varphi} x^{2} R_{\varphi} \ldots R_{\varphi} x^{m} R_{\varphi} x^{1}$. Let $C$ 


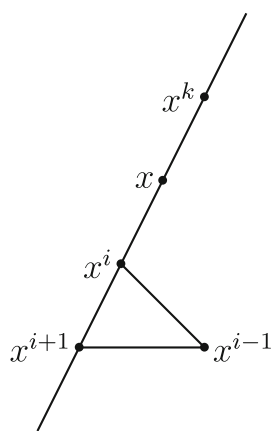

(i)

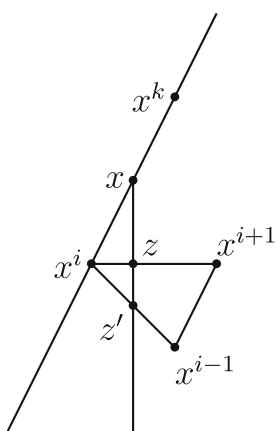

(ii)

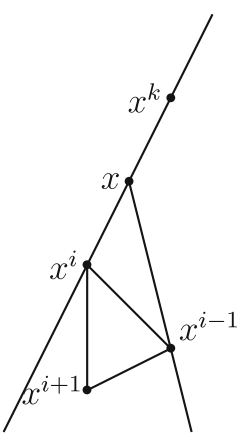

(iii)

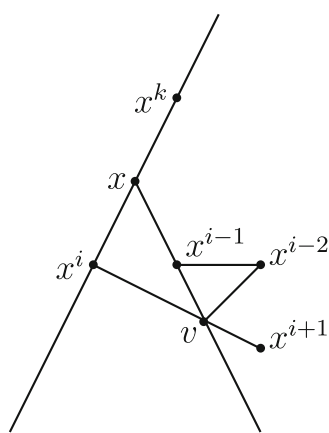

(iv)

Fig. 3 The four cases in the proof of Theorem 4.5

be the convex hull of these $m$ points, and let $x:=\varphi(C)$. Then $x \in \operatorname{bd}(C)$, and by IIA, there are $x^{i}$ and $x^{k}$ with $i, k \in\{1, \ldots, m\}$ and $k \notin\{(i-1) \bmod m, i,(i+1) \bmod m\}$ such that $x \in\left(x^{i}, x^{k}\right)$ and $\left(x^{i}, x^{k}\right) \cap\left\{x^{1}, \ldots, x^{m}\right\}=\emptyset$. We now consider four cases concerning the location of $x^{i+1}$. (For simplicity of notation we write $i+1$ and $i-1$ instead of $(i+1) \bmod m$ and $(i-1) \bmod m$.) See Fig. 3, where these four cases are illustrated.

Case (i): $x^{i+1}$ is a point on the line through $x^{i}$ and $x^{k}$. In this case $x^{i+1} \in\left[x, x^{i}, \rightarrow\right)$, and in particular $x^{i} \in\left[x, x^{i+1}\right]$, since otherwise $x^{k} R_{\varphi} x^{i+1}$ by Theorem 3.4, a contradiction. Then for every point $y \in\left[x^{i-1}, x^{i+1}\right] \cup\left[x^{i+1}, x^{i}\right]$ we have $[x, y] \cap\left[x^{i-1}, x^{i}\right] \neq$ $\emptyset$, hence, by Theorem 3.4, if $\hat{y}$ is the point of intersection then $\hat{y} R_{\varphi} y$. This implies that $\varphi\left(\operatorname{conv}\left(\left\{x^{i-1}, x^{i}, x^{i+1}\right\}\right)\right)$ must be a point of $\left[x^{i-1}, x^{i}\right]$, and hence, by IIA, $\varphi\left(\operatorname{conv}\left(\left\{x^{i-1}, x^{i}, x^{i+1}\right\}\right)\right)=x^{i-1}$. This, however, implies $x^{i-1} R_{\varphi} x^{i+1}$, so that we obtain a cycle of length $(m-1)$ by dropping the point $x^{i}$ from the original cycle of length $m$. This contradicts the induction hypothesis.

Case (ii): $x^{i+1}$ is not a point on the line through $x^{i}$ and $x^{k}$, and the half-line $\left[x^{i}, x^{i+1}, \rightarrow\right)$ is in the convex hull of the half-lines $\left[x^{i}, x, \rightarrow\right)$ and $\left[x^{i}, x^{i-1}, \rightarrow\right)$. (Observe that $x^{i} \notin\left[x, x^{i-1}\right]$ since otherwise $x^{i} R_{\varphi} x^{i-1}$ by Theorem 3.4, a contradiction.) In this case, consider a half-line $\ell$ starting from $x$ and intersecting the segments $\left[x, x^{i+1}\right]$ and $\left[x, x^{i-1}\right]$ in points $z$ and $z^{\prime}$, respectively. Then $z R_{\varphi} z^{\prime}$ by Theorem 3.4 , $z^{\prime} R_{\varphi} x^{i}$ by Theorem 3.4 since $x^{i-1} R_{\varphi} x^{i}$, and $x^{i} R_{\varphi} z$ by Theorem 3.4 since $x^{i} R_{\varphi} x^{i+1}$. Hence, we have a cycle of length three, which is a contradiction to Lemma 4.4.

Case (iii): $x^{i+1}$ is not a point on the line through $x^{i}$ and $x^{k}$, and the half-line $\left[x^{i}, x^{i-1}, \rightarrow\right)$ is in the convex hull of the half-lines $\left[x^{i}, x, \rightarrow\right)$ and $\left[x^{i}, x^{i+1}, \rightarrow\right)$; and $x^{i+1}$ is separated from $x^{k}$ by the line through $x$ and $x^{i-1}$. (Observe that $x^{i-1} \notin$ $\left[x, x^{k}, \rightarrow\right.$ ) since otherwise $x^{i-1} R_{\varphi} x^{k}$ by Theorem 3.4, a contradiction.) The proof for this case is identical to the proof of case (i).

Case (iv): $x^{i+1}$ is not a point on the line through $x^{i}$ and $x^{k}$, and the half-line $\left[x^{i}, x^{i-1}, \rightarrow\right)$ is in the convex hull of the half-lines $\left[x^{i}, x, \rightarrow\right)$ and $\left[x^{i}, x^{i+1}, \rightarrow\right)$; and $x^{i+1}$ and $x^{k}$ are on the same side of the line through $x$ and $x^{i-1}$. To deal with this case, let $v$ be the point of intersection of $\left[x^{i}, x^{i+1}\right]$ and $\left[x, x^{i-1}, \rightarrow\right)$. By Theorem 3.4, $v R_{\varphi} x^{i+1}$. Consider the point $x^{i-2}$ (recall that $m \geq 4$ ). Since 
for any point $y \in\left[x^{i-1}, v\right] \cup\left[v, x^{i-2}\right]$ the line segment $[x, y]$ intersects the line segment $\left[x^{i-2}, x^{i-1}\right]$ in some point $\hat{y}$, Theorem 3.4 implies $\hat{y} R_{\varphi} y$ for any such point $y$. Hence $\varphi\left(\operatorname{conv}\left(\left\{x^{i-2}, x^{i-1}, v\right\}\right)\right) \in\left[x^{i-2}, x^{i-1}\right]$, and therefore IIA implies $\varphi\left(\operatorname{conv}\left(\left\{x^{i-2}, x^{i-1}, v\right\}\right)\right)=x^{i-2}$. We now have $\ldots R_{\varphi} x^{i-2} R_{\varphi} v R_{\varphi} x^{i+1} R_{\varphi} \ldots$, hence a cycle of length $m-1$. This contradiction takes care of case (iv) and completes the proof of the proposition.

Unfortunately, Theorem 4.5 does not extend to higher dimensions. This can be shown by a modification of the extension of an example of Gale (1960) for consumer theory in Peters and Wakker (1991); see also Bossert (1994). Peters and Wakker (1991) consider, for $k=3$, the subset $\Sigma \subseteq \mathcal{C}$, consisting of all elements of $C \in \mathcal{C}$ that satisfy (i) $x \geq \mathbf{0}$ for all $x \in C$; (ii) $y \in C$ for all $x, y \in \mathbb{R}^{3}$ with $x \in C$ and $\mathbf{0} \leq y \leq x$; and (iii) $x>\mathbf{0}$ for all $x \in \mathrm{PO}(C)$. Here, $\mathrm{PO}(C):=\{x \in C \mid \forall y \in C, y \geq x \Rightarrow y=x\}$ is the Pareto optimal subset of $C$. ${ }^{5}$ Then a choice function $\varphi: \Sigma \rightarrow \mathbb{R}^{3}$ is constructed that satisfies IIA and is continuous (and Pareto optimal), but admits a cycle of length four. It can be verified that this construction is invariant under a translation over a vector with equal coordinates. More precisely, let $\mathbf{1}:=(1,1,1) \in \mathbb{R}^{3}$. Then, for any sets $C, C^{\prime} \in \Sigma$ such that there is a number $\alpha \in \mathbb{R}$ with $\mathrm{PO}(C)=\left\{x+\alpha \mathbf{1} \mid x \in \mathrm{PO}\left(C^{\prime}\right)\right\}$, it holds that $\varphi(C)=\varphi\left(C^{\prime}\right)+\alpha \mathbf{1}$. Therefore, $\varphi$ can be extended to all of $\mathcal{C}$ while preserving IIA and CON as follows. For any set $C^{\prime} \in \mathcal{C}$, take a number $\alpha \in \mathbb{R}$ such that $C:=\left\{x+\alpha \mathbf{1} \mid x \in C^{\prime}\right\}$ satisfies (i) and (iii) above, and extend this set $C$ to a set $\hat{C}:=\left\{x \in \mathbb{R}^{3} \mid \exists y \in C\right.$ such that $\left.\mathbf{0} \leq x \leq y\right\}$. Then $\hat{C} \in \Sigma$. Now define $\varphi\left(C^{\prime}\right):=\varphi(\hat{C})-\alpha \mathbf{1}$

\section{Representability}

As mentioned earlier, the one-dimensional case is special because rationalizabilityrepresentability is guaranteed; see the discussion in Sect. 3. Furthermore, the previous section has established that, in higher dimensions, IIA is, in general, not sufficient for rationalizability even in the presence of CON. Therefore, in order to obtain rationalizability-representability, we strengthen IIA to the acyclicity of $R_{\varphi}$-that is, the strong axiom of revealed preference. In this case, $\overline{R_{\varphi}}$ (or any of its extensions; see Szpilrajn 1930; Richter 1966) can be used as a rationalization.

The continuity property CIC is not sufficient to guarantee the rationalizabilityrepresentability of $\varphi$ if added to the acyclicity of $R_{\varphi}$. This is established in the following example.

Example 5.1 Let $k=2$ and define the relation $R$ on $\mathbb{R}^{2}$ by

$$
x R y: \Leftrightarrow\left[\left|x_{1}\right|<\left|y_{1}\right|\right] \text { or }\left[\left|x_{1}\right|=\left|y_{1}\right| \text { and }\left|x_{2}\right| \leq\left|y_{2}\right|\right]
$$

for all $x, y \in \mathbb{R}^{2}$. This is a single-peaked choice function with peak $p=\mathbf{0}$. Now let, for all $C \in \mathcal{C}, \varphi(C)$ be the unique best element in $C$ according to $R$. This choice function is well-defined because $C$ is non-empty, compact and convex. Thus, $\varphi$ is rationalizable

\footnotetext{
5 Of course, in our context Pareto optimality has no special appeal.
} 
and $R_{\varphi}$ is equal to $R$ and therefore acyclic (which, of course, also implies IIA). CIC is satisfied but CON is not. Because of the lexicographic nature of this example, $\varphi$ is not rationalizable-representable.

To obtain a representation theorem, we assume below that $R_{\varphi}$ is acyclic and $\varphi$ satisfies CON. We first establish two preliminary results. For $x \in \mathbb{R}^{k}$ and $\varepsilon>0$, we use $\mathcal{B}(x, \varepsilon)$ to denote the open $\varepsilon$-ball around $x$, that is, the set of points in $\mathbb{R}^{k}$ that have Euclidean distance smaller than $\varepsilon$ to $x$.

Lemma 5.2 Let $k \geq 2$, let $x, z \in \mathbb{R}^{k}$ be such that $x \neq z$ and $z R_{\varphi} x$ and let $y \in$ $[z, x, \rightarrow) \backslash[z, x]$. If choice function $\varphi: \mathcal{C} \rightarrow \mathbb{R}^{k}$ satisfies IIA and CON, then there is an $\varepsilon>0$ such that $x R_{\varphi} v$ for all $v \in \mathcal{B}(y, \varepsilon)$.

Proof By CON, there is a $\delta>0$ such that $\varphi([w, x]) \in[w, x)$ for all $w \in \mathcal{B}(z, \delta)$. By Theorem 3.4, $x R_{\varphi} v$ for all $w \in \mathcal{B}(z, \delta)$ and $v \in[w, x, \rightarrow) \backslash[w, x]$. We can choose $\varepsilon>0$ sufficiently small so that, for each $v \in \mathcal{B}(y, \varepsilon)$, there is a $w \in \mathcal{B}(z, \delta)$ such that $v \in[w, x, \rightarrow) \backslash[w, x]$. Then $x R_{\varphi} v$ for all $v \in \mathcal{B}(y, \varepsilon)$.

Lemma 5.3 Let $k \geq 2$ and let $x, w \in \mathbb{R}^{k}$ be such that $x \neq w$ and $x R_{\varphi} w$. If choice function $\varphi: \mathcal{C} \rightarrow \mathbb{R}^{\bar{k}}$ satisfies IIA and CON, then there is an $a \in \mathbb{Q}^{k} \backslash\{x, w\}$ such that $x \overline{R_{\varphi}} a R_{\varphi} w$.

Proof By CON, there is a $\delta>0$ such that $\|\varphi([v, w])-x\|<\|x-w\| / 3$ for all $v \in \mathcal{B}(x, \delta)$.

If there is no $z \in \mathbb{R}^{k} \backslash\{x\}$ with $z R_{\varphi} x$, then $x$ is a peak of $\varphi$ and we choose $y \in \mathcal{B}(x, \delta)$ arbitrarily. Otherwise, let $z \in \mathbb{R}^{k} \backslash\{x\}$ with $z R_{\varphi} x$ and choose $y \in \mathcal{B}(x, \delta) \cap[z, x, \rightarrow)$ $\backslash[z, x]$. By Lemma 5.2, we can choose $\varepsilon>0$ such that $\mathcal{B}(y, \varepsilon) \subseteq \mathcal{B}(x, \delta)$ and $x R_{\varphi} v$ for all $v \in \mathcal{B}(y, \varepsilon)$. Since the set $\left\{[v, w] \in \mathbb{R}^{k} \mid v \in \mathcal{B}(y, \varepsilon)\right\}$ has full dimension, we can take a $\bar{v} \in \mathcal{B}(y, \varepsilon)$ such that $[\bar{v}, w]$ contains a point $a \in \mathbb{Q}^{k} \backslash\{w\}$ with $\|a-w\|<$ $\|x-w\| / 3$. Since $\|\varphi([\bar{v}, w])-x\|<\|x-w\| / 3$ and $\|a-w\|<\|x-w\| / 3$, Theorem 3.4 implies $\varphi([\bar{v}, w]) R_{\varphi} a$ and $a R_{\varphi} w$. Since $\bar{v} \in \mathcal{B}(x, \delta), x R_{\varphi} \varphi([\bar{v}, w])$. So $x \overline{R_{\varphi}} a R_{\varphi} w$.

We now obtain our representation result.

Theorem 5.4 Let $k \geq 2$. If a choice function $\varphi: \mathcal{C} \rightarrow \mathbb{R}^{k}$ satisfies $C O N$ and is such that $R_{\varphi}$ is acyclic, then $\varphi$ is rationalizable-representable.

Proof The acyclicity of $R_{\varphi}$ implies that $\varphi$ is rationalizable and $\overline{R_{\varphi}}$ is a rationalization of $\varphi$. We complete the proof by establishing the existence of a representation $u$ of $\overline{R_{\varphi}}$.

Lemma 5.3 straightforwardly implies

$x \neq y \quad$ and $x \overline{R_{\varphi}} y \Rightarrow$ there exists an $a \in \mathbb{Q}^{k} \backslash\{x, y\}$ such that $x \overline{R_{\varphi}} a \overline{R_{\varphi}} y$

for all $x, y \in \mathbb{R}^{k}$. Let $\mathbb{Q}^{k}=\left\{a_{1}, a_{2}, \ldots\right\}$ be an enumeration of the (countable) set $\mathbb{Q}^{k}$. Define $u: \mathbb{R}^{k} \rightarrow \mathbb{R}$ by

$$
u(x)=\sum_{k \in \mathbb{N} \mid x \overline{R_{\varphi}} a_{k}} 2^{-k}
$$


for all $x \in \mathbb{R}^{k}$. By (1) and the antisymmetry and transitivity of $\overline{R_{\varphi}}, u$ represents $\overline{R_{\varphi}} \cdot 6$

The reason why CIC is sufficient for a representation result in the one-dimensional case but not for higher dimensions is quite intuitive. In the one-dimensional case, variations along a straight line are sufficient to span a full-dimensional neighborhood of a point but, of course, this is not the case in higher dimensions.

A property that is often associated with generalizations of single-peaked preferences to higher dimensions is strict quasi-concavity. Our final result establishes that if a representation of $\overline{R_{\varphi}}$ exists and CIC is satisfied, then this function is strictly quasiconcave.

Theorem 5.5 Let $k \geq 2$. If a choice function $\varphi: \mathcal{C} \rightarrow \mathbb{R}^{k}$ satisfies $C I C$ and a function $u: \mathbb{R}^{k} \rightarrow \mathbb{R}$ represents $\overline{R_{\varphi}}$, then $u$ is strictly quasi-concave.

Proof First note that $\varphi$ satisfies IIA, and thus, by Theorem 3.4, is single-peaked. Let $z, z^{\prime} \in \mathbb{R}^{k}$, let $0<\alpha<1$ and $z^{\prime \prime}:=\alpha z+(1-\alpha) z^{\prime}$. Let $y=\varphi\left(\left[z, z^{\prime}\right]\right)$, then $y R_{\varphi} z$ and $y R_{\varphi} z^{\prime}$. Since $\varphi$ is single-peaked, Proposition 3.2 implies $z^{\prime \prime} R_{\varphi} z$ or $z^{\prime \prime} R_{\varphi} z^{\prime}$. Moreover, since $R_{\varphi}$ is antisymmetric, this implies $u\left(z^{\prime \prime}\right)>\min \left\{u(z), u\left(z^{\prime}\right)\right\}$. This proves strict quasi-concavity of $u$.

\section{Concluding remarks}

Single-peaked preferences play an important role in the economics and politicalscience literatures. In this paper, we have examined single-peakedness from a choicetheoretic perspective, thus providing additional perspectives on the foundations of this notion. In particular, we have characterized a class of single-peaked choice functions by contraction consistency (IIA) and (collinear) continuity. For this class-under collinear or full continuity-we have obtained detailed results on rationalizability and representability. Both continuity and IIA are generally accepted conditions. IIA generalizes the classical weak axiom of revealed preference and, therefore, captures the rationality of the decision maker. Thus, our results give strong support to single-peaked choice.

We have assumed the domain of all non-empty compact convex sets in this paper, but it is not difficult to check, by going over the proofs, that all our results would hold on some smaller domains as well, e.g., the domain of all polytopes. Most of our results even go through on the minimal domain of line segments, i.e., convex hulls of two points. Only for the positive results on rationalizability in Lemma 4.4 and Theorem 4.5 this is not clear, since their proofs use sets that are the convex hulls of more than two points.

\footnotetext{
6 This argument, using (1), is a variation on Lemma II in Debreu (1954) for partial orders. See also Jaffray (1975).
} 


\section{Appendix: Proof of Theorem 4.2}

Let $n \in \mathbb{N}$ with $n \geq 3$ and consider a convex $n$-polygon $\mathcal{P}$ in $\mathbb{R}^{2}$ with consecutive vertices $x^{1}, \ldots, x^{n}$. This means that $\mathcal{P}$ is the convex hull of $\left\{x^{1}, \ldots, x^{n}\right\}$, every $x^{i}$ is an extreme point, and the boundary is the union of the line segments $\left[x^{i}, x^{i+1}\right]$ for $i=1, \ldots, n$ with $x^{n+1}:=x^{1}$. A point $\hat{x} \in \mathcal{P}$ is called a Brocard point ${ }^{7}$ if $\measuredangle \hat{x} x^{1} x^{2}=\measuredangle \hat{x} x^{2} x^{3}=\cdots=\measuredangle \hat{x} x^{n-1} x^{n}=\measuredangle \hat{x} x^{n} x^{1}$. Denote this common angle size by $\alpha_{\hat{x}}$.

Denote by $O(C)$ the area of a set $C$ in $\mathbb{R}^{2}$. For points $x, y, z \in \mathbb{R}^{2}$ denote by $\triangle(x y z)$ the triangle with vertices $x, y$, and $z$. Recall that $A(n)=\frac{n-2}{2 n} \pi$ is equal to $(1 / 2) \measuredangle x^{1} x^{2} x^{3}$ if $\mathcal{P}$ is a regular polygon.

Theorem 1 Let $\hat{x}$ be a Brocard point in $\mathcal{P}$ with common angle size $\alpha_{\hat{x}}$. Then

(i) $\cot \alpha_{\hat{x}}=\left(\sum_{i=1}^{n}\left\|x^{i+1}-x^{i}\right\|^{2}\right) / 4 O(\mathcal{P})$;

(ii) $\alpha_{\hat{x}} \leq A(n)$.

Proof For any triangle $\triangle(x y z)$ we have the familiar formula $O(\triangle(x y z))=(1 / 2) \| y-$ $x\|\cdot\| z-x \| \sin \measuredangle y x z$. Hence,

$$
\begin{aligned}
O(\mathcal{P})= & O\left(\triangle\left(\hat{x} x^{1} x^{2}\right)\right)+O\left(\triangle\left(\hat{x} x^{2} x^{3}\right)\right)+\cdots+O\left(\triangle\left(\hat{x} x^{n} x^{1}\right)\right) \\
= & (1 / 2)\left\|x^{1}-\hat{x}\right\| \cdot\left\|x^{2}-x^{1}\right\| \sin \measuredangle \hat{x} x^{1} x^{2} \\
& +(1 / 2)\left\|x^{2}-\hat{x}\right\| \cdot\left\|x^{3}-x^{2}\right\| \sin \measuredangle \hat{x} x^{2} x^{3} \\
& +\cdots+(1 / 2)\left\|x^{n}-\hat{x}\right\| \cdot\left\|x^{1}-x^{n}\right\| \sin \measuredangle \hat{x} x^{1} x^{2} \\
= & (1 / 2) \sin \alpha_{\hat{x}}\left[\left\|x^{1}-\hat{x}\right\| \cdot\left\|x^{2}-x^{1}\right\|+\left\|x^{2}-\hat{x}\right\| \cdot\left\|x^{3}-x^{2}\right\|\right. \\
& \left.+\cdots+\left\|x^{n}-\hat{x}\right\| \cdot\left\|x^{1}-x^{n}\right\|\right]
\end{aligned}
$$

hence

$$
\sin \alpha_{\hat{x}}=\frac{2 O(\mathcal{P})}{\sum_{i=1}^{n}\left\|x^{i}-\hat{x}\right\| \cdot\left\|x^{i+1}-x^{i}\right\|}
$$

For any triangle $\triangle(x y z)$ we moreover have the familiar formula

$$
\cos \measuredangle y x z=\frac{\|y-x\|^{2}+\|z-x\|^{2}-\|y-z\|^{2}}{2\|y-x\| \cdot\|z-x\|} .
$$

Hence

$$
\cos \alpha_{\hat{x}}=\frac{\left\|x^{i+1}-x^{i}\right\|^{2}+\left\|\hat{x}-x^{i}\right\|^{2}-\left\|x^{i+1}-\hat{x}\right\|^{2}}{2\left\|x^{i+1}-x^{i}\right\| \cdot\left\|\hat{x}-x^{i}\right\|}
$$

\footnotetext{
7 See Honsberger (1995) or Weisstein (2005).
} 
for all $i \in\{1, \ldots, n\}$. By adding these expressions for all $i=1, \ldots, n$, we obtain

$$
\cos \alpha_{\hat{x}}=\frac{\sum_{i=1}^{n}\left\|x^{i+1}-x^{i}\right\|^{2}}{2 \sum_{i=1}^{n}\left\|x^{i}-\hat{x}\right\| \cdot\left\|x^{i+1}-x^{i}\right\|} .
$$

Combining (2) and (3), we obtain

$$
\cot \alpha_{\hat{x}}=\frac{\cos \alpha_{\hat{x}}}{\sin \alpha_{\hat{x}}}=\left(\sum_{i=1}^{n}\left\|x^{i+1}-x^{i}\right\|^{2}\right) / 4 O(\mathcal{P}),
$$

which proves (i).

For (ii), note that $\alpha_{\hat{x}}$ is maximal if its cotangent value is minimal. Among polygons of fixed circumference, a regular $n$-polygon minimizes the sum of the squares of the edges (since this sum-function is convex) and maximizes the area. ${ }^{8}$ Hence, by (i), $\alpha_{\hat{x}}$ is maximal for a regular $n$-polygon. This implies (ii).

We now prove Theorem 4.2, which is a slight extension of Theorem 1.

Proof of Theorem 4.2 Consider the half-line $\ell=\left[x^{2}, x^{1}, \rightarrow\right)$ through $x^{1}$ starting from $x^{2}$ and the half-line $\ell^{\prime}=\left[x^{n-1}, x^{n}, \rightarrow\right)$ through $x^{n}$ starting from $x^{n-1}$.

If $\ell$ and $\ell^{\prime}$ do not intersect, then we can find a point $z \notin \mathcal{P}$ on $\ell^{\prime}$ sufficiently far from $x^{n}$ such that $\measuredangle \hat{x} z x^{1}<\alpha$. Since $\measuredangle \hat{x} x^{n} x^{1} \geq \alpha$, by a continuity consideration there must be a point $y \in\left[x^{n}, z\right]$ such that $\measuredangle \hat{x} y x^{\overline{1}}=\alpha$. Then $\hat{x}$ is a Brocard point in the convex $n$-polygon with vertices $x^{1}, \ldots, x^{n-1}, y$ with $\alpha_{\hat{x}}=\alpha$. By Theorem A(ii), $\alpha \leq A(n)$.

If $\ell$ and $\ell^{\prime}$ intersect, say in some point $z$, then it is easy to see that $\measuredangle \hat{x} z x^{1}<\alpha$. The argument continues as in the first case.

Open Access This article is distributed under the terms of the Creative Commons Attribution Noncommercial License which permits any noncommercial use, distribution, and reproduction in any medium, provided the original author(s) and source are credited.

\section{References}

Ballester, M., Haeringer, G.: A characterization of single-peaked preferences. Unpublished manuscript, Universitat Autònoma de Barcelona (2006)

Barberà, S., Gul, F., Stacchetti, E.: Generalized median voting schemes and committees. J Econ Theory 61, 262-289 (1993)

Barberà, S., Jackson, M.: A characterization of strategy-proof social choice functions for economies with pure public goods. Soc Choice Welfare 11, 241-252 (1994)

Black, D.: On the rationale of group decision-making. J Polit Econ 56, 23-34 (1948)

Bossert, W.: Rational choice and two-person bargaining solutions. J Math Econ 23, 549-563 (1994)

Debreu, G.: Representation of a preference ordering by a numerical function. In: Thrall, R., Coombs, C., Davies, R. (eds.) Decision Processes, pp. 159-175. New York: Wiley (1954)

Debreu, G.: Smooth preferences. Econometrica 40, 603-615 (1972)

Dutta, B., Peters, H., Sen, A.: Strategy-proof probabilistic mechanisms in economies with pure public goods. J Econ Theory 106, 392-416 (2002)

\footnotetext{
8 This is a classical result known as the Isoperimetric Theorem, cf. Weisstein (2006).
} 
Ehlers, L., Storcken, T.: Arrow's theorem in spatial environments. Unpublished manuscript, Université de Montréal (2002)

Gale, D.: A note on revealed preference. Economica 27, 348-354 (1960)

Honsberger, R.: The Brocard Angle. Section 10.2 in: Episodes in Nineteenth and Twentieth Century Euclidean Geometry, Mathematical Association of America, Washington, DC, pp. 101-106 (1995)

Inada, K.: The simple majority rule. Econometrica 37, 490-506 (1969)

Jaffray, J.-Y.: Semicontinuous extension of a partial order. J Math Econ 2, 395-406 (1975)

Le Breton, M., Weymark, J.: Arrovian social choice theory on economic domains. In: Arrow, K., Sen, A., Suzumura, K. (eds.) Handbook of Social Choice and Welfare, vol. 2. Amsterdam: Elsevier (2007, forthcoming)

Moulin, H.: On strategy-proofness and single-peakedness. Public Choice 35, 437-455 (1980)

Moulin, H.: Generalized Condorcet-winners for single peaked and single-plateau preferences. Soc Choice Welfare 1, 127-147 (1984)

Nash, J.: The bargaining problem. Econometrica 18, 155-162 (1950)

Peters, H., Wakker, P.: Independence of irrelevant alternatives and revealed group preferences. Econometrica 59, 1787-1801 (1991)

Peters, H., Wakker, P.: WARP does not imply SARP for more than two commodities. J Econ Theory 62, 152-160 (1994)

Richter, M.: Revealed preference theory. Econometrica 41, 1075-1091 (1966)

Richter, M.: Rational choice. In: Chipman, J., Hurwicz, L., Richter, M. Sonnenschein, H. (eds.) Preferences, Utility, and Demand, pp. 29-58. New York: Harcourt Brace Jovanovich (1971)

Sen, A.: Collective choice and social welfare. San Francisco: Holden-Day (1970)

Sprumont, Y.: The division problem with single-peaked preferences: a characterization of the uniform allocation rule. Econometrica 59, 509-519 (1991)

Szpilrajn, E.: Sur l'extension de l'ordre partiel. Fundam Math 16, 386-389 (1930)

Weisstein, E.W.: Brocard Angle. From: MathWorld-A Wolfram Web Resource. http://mathworld.wolfram. com/BrocardAngle.html (2005)

Weisstein, E.W.: Isoperimetric Theorem. From: MathWorld-A Wolfram Web Resource. http://mathworld. wolfram.com/IsoperimetricTheorem.html (2006) 